\title{
Uncommon mandibular osteomyelitis in a cat caused by Nocardia africana
}

\author{
Marconi Rodrigues de Farias ${ }^{1}$, Juliana Werner ${ }^{1}$, Márcio Garcia Ribeiro ${ }^{2,5^{*}}$, Sabrina Marin Rodigheri ${ }^{3}$, \\ Carolina Zaghi Cavalcante ${ }^{1}$, Kung Darh Chi ${ }^{1}$, Larissa Anuska Zeni Condas ${ }^{2}$, Tohru Gonoi ${ }^{4}$, \\ Tetsuhiro Matsuzama ${ }^{4}$ and Katsukiyo Yazama ${ }^{4}$
}

\begin{abstract}
Background: Nocardiosis is an unusual infection in companion animals characterized by suppurative to pyogranulomatous lesions, localized or disseminated. Cutaneous-subcutaneous, pulmonary and systemic signs are observed in feline nocardiosis. However, osteomyelitis is a rare clinical manifestation in cats. Nocardia cyriacigeorgica (formerly N. asteroides sensu stricto), Nocardia brasiliensis, Nocardia otitidiscaviarum, and Nocardia nova are the most common pathogenic species identified in cats, based on recent molecular classification (16S rRNA gene). The present report is, to our knowledge, the first case of mandibular osteomyelitis in a cat caused by Nocardia africana, diagnosed based upon a combination of methods, including molecular techniques.

Case presentation: A one-year-old non-neutered female cat, raised in a rural area, was admitted to the Companion Animal Hospital-PUCPR, São José dos Pinhais, State of Paraná, Brazil, with a history a progressive facial lesion, difficulty apprehending food, loss of appetite, apathy and emaciation. Clinical examination showed fever, submandibular lymphadenitis, and a painless, $8 \mathrm{~cm}$ diameter mass, which was irregularly-shaped, of firm consistency, and located in the region of the left mandible. The skin around the lesion was friable, with diffuse inflammation (cellulitis), multiple draining sinuses, and exudation of serosanguinous material containing whitish "sulfur" granules.

Diagnosis was based initially in clinical signs, microbiological culture, cytological, and histopathological findings, and radiographic images. Molecular sequencing of $16 \mathrm{~S}$ rRNA of isolate allowed diagnosis of Nocardia africana. Despite supportive care and antimicrobial therapy based on in vitro susceptibility testing the animal died.

Conclusion: The present report describes a rare clinical case of feline osteomyelitis caused by Nocardia africana, diagnosed based upon a combination of clinical signs, microbiological culture, cytological and histopathological findings, radiographic images, and molecular methods. The use of modern molecular techniques constitutes a quick and reliable method for Nocardia species identification, and may contribute to identification to new species of Nocardia that are virulent in cats.
\end{abstract}

Keywords: Cat, Nocardia africana, Feline nocardiosis, Osteomyelitis

\footnotetext{
* Correspondence: mgribeiro@fmvz.unesp.br

${ }^{2}$ Department of Veterinary Hygiene and Public Health, School of Veterinary

Medicine and Animal Sciences, Univ. Estadual Paulista-UNESP, Botucatu, Sao

Paulo, Brazil

${ }^{5}$ Infectious Diseases of Domestic Animals, Department of Veterinary Hygiene and Public Health, School of Veterinary Medicine and Animal Sciences, Univ. Estadual Paulista-UNESP, Code 18618-970, POBox 560, Botucatu, Sao Paulo, Brazil

Full list of author information is available at the end of the article
}

\section{Biomed Central}

(C) 2012 de Farias et al.; licensee BioMed Central Ltd. This is an Open Access article distributed under the terms of the Creative Commons Attribution License (http://creativecommons.org/licenses/by/2.0), which permits unrestricted use, distribution, and reproduction in any medium, provided the original work is properly cited. 


\section{Background}

Nocardiosis is an opportunistic disease for humans and domestic animals, caused by aerobic actinomycetes from the genus Nocardia. These organisms are ubiquitous, soil saprophytes, and are found in organic material, water and plants.

In companion animals, nocardial infection is considered rare, and are characterized by suppurative to pyogranulomatous lesions, localized or disseminated [1].

Routine diagnosis in companion animals is based on microbiological culture, and phenotypic characterization [2]. Currently, species of the genus Nocardia were reclassified using molecular methods. Nocardia cyriacigeorgica (formerly $N$. asteroides sensu stricto), Nocardia brasiliensis, Nocardia otitidiscaviarum, and Nocardia nova complex are the most pathogenic species involved in feline nocardiosis [3].

The transmission of feline nocardiosis is intimately related with the inhalation of soil organisms, and traumatic inoculation through wounds. Immunosuppressive diseases, such as immunodeficiency and leukemia in cats have been reported concurrently with feline nocardiosis [4]. Clinically, feline nocardiosis is characterized by cutaneous-subcutaneous lesions (abscesses, mycetoma, cellulitis), with draining sinuses, and less frequently by pulmonary and systemic forms, such as peritonitis and encephalitis [3].

Currently, human nocardiosis has emerged as an increasingly common opportunistic pathogen among immunosuppressed patients, particularly those infected by acquired immune deficiency syndrome-AIDS, other diseases (tuberculosis, lymphoma, lymphosarcoma, cirrhosis, diabetes), or debilitating conditions (organ transplantation, alcoholism, or prolonged use of corticosteroids) [3,5].

The present report describes a rare clinical case of osteomyelitis in a cat caused by Nocardia africana.

\section{Case presentation}

A one-year-old non-neutered female cat, raised in a rural area, was admitted to the Companion Animal Hospital-PUCPR, São José dos Pinhais, State of Paraná, Brazil. The cat had a history a progressive facial lesion, difficulty apprehending food, loss of appetite, apathy and emaciation for approximately five months. The animal had no history of lesions in the mandibular region or mouth. Clinical examination showed fever, submandibular lymphadenitis and a painless, $8 \mathrm{~cm}$ diameter mass, which was irregular-shaped, of firm consistency, and located in the region of the left mandible (Figure 1). The skin around the lesion was friable, with diffuse inflammation (cellulitis), multiple draining sinuses, and exudation of serosanguinous material containing whitish "sulfur" granules. A blood sample for a complete blood count $(\mathrm{CBC})$ was taken. Hematologic abnormalities

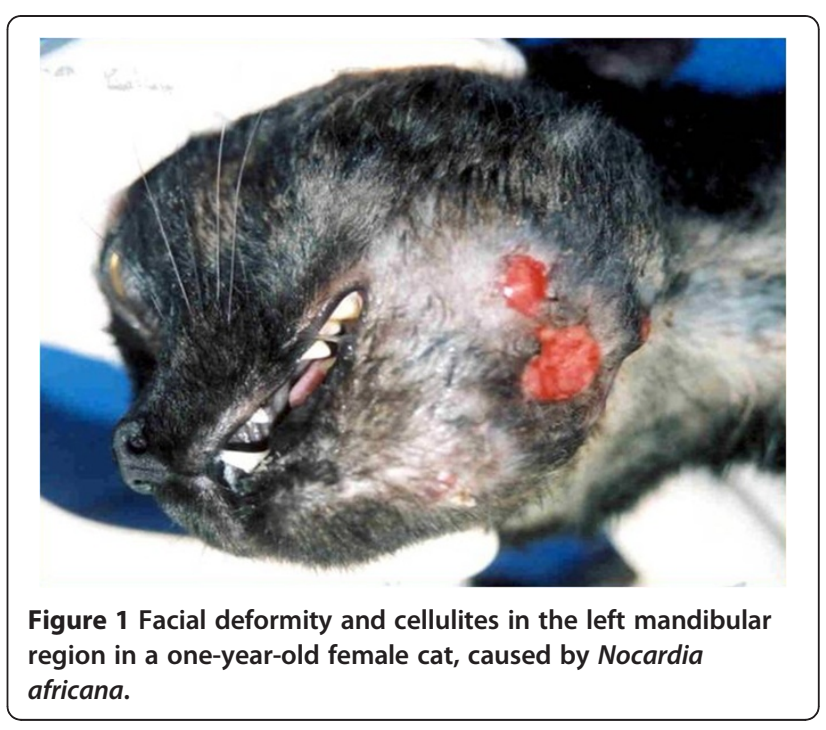

included leukocytosis $(21,400 / \mu \mathrm{L}$, reference interval [RI] $6,000-17,000 / \mu \mathrm{L})$, neutrophilia $(18,200 / \mu \mathrm{L}$, RI 3,000$11,500 / \mu \mathrm{L})$, monocytosis $(2,640 / \mu \mathrm{L}$, RI $200-1,400 / \mu \mathrm{L})$, and normocytic, normochromic, non-regenerative anemia (RBC $1.2 \times 10^{6} / \mu \mathrm{L}$, RI 5.5-8.5 x10 $/ \mu \mathrm{L}$; HCT $18 \%$, RI $37-$ $55 \%)$. Biochemical tests for renal and hepatic function were normal. Serological tests for feline immunodeficiency virus (FIV) and feline leukemia virus (FeLV) were negative (Snap FIV Antibody/FeLV Antigen Combo Test - IDEXX Laboratories, USA).

Radiography of the mandible showed intense bone proliferation and osteolysis (Figure 2). Initially, fine needle aspiration was performed in the lesion [5] and sample was submitted simultaneously for cytological analysis and microbiological culture. Sample obtained by fine needle aspiration was stained with Gram and Panoptic stains, and showed numerous gram-positive, filamentous branching organisms. Furthermore, biopsy of lesion was taken. This fragment was stained with haemathoxilin-

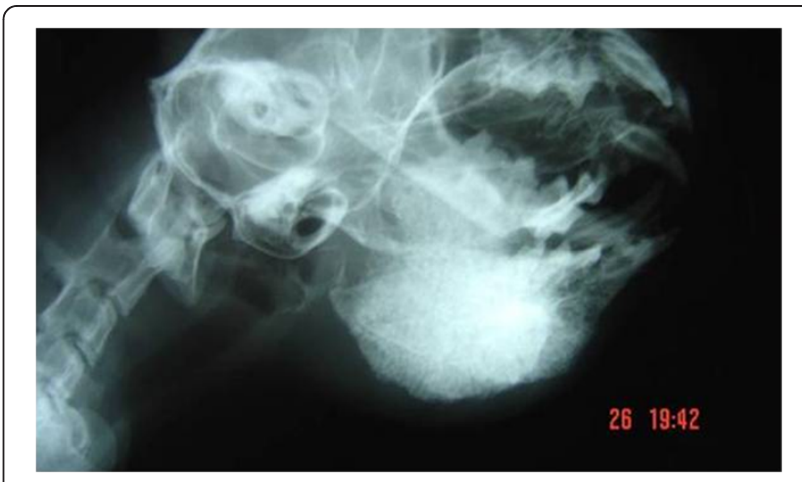

Figure 2 Facial radiograph of the mandibular region in a cat with osteomyelitis caused by Nocardia africana. Note severe osteolysis and bony proliferation in the mandible. 
eosin and revealed a central region of necrosis and suppuration containing filamentous organisms, surrounded by neutrophils, macrophages, epithelioid cells and fibroblasts. Specimens were cultured on sheep blood agar and Sabouraud agar, incubated aerobically at $37^{\circ} \mathrm{C}$ for 72-96 h. After 72 h, numerous whitish-orange, circular, convex, dry, with powdery surface colonies were observed in sheep blood agar and Sabouraud agar (Figure 3). From these colonies, gram-positive, partially acid-fast (modified Ziehl-Neelsen), filamentous microorganisms were observed, suggestive of the genus Nocardia [2].

Classical biochemical tests based on casein, xanthine, hypoxanthine, and tyrosine assimilation were carried out, as well use of other substrates in a commercial kit (API 20C AUX strips, BioMeriex, Hazelwood, MO.) [6]. These tests allowed the preliminary phenotypic classification of isolate as Nocardia nova complex [7].

Molecular analysis of the isolate was based on a subunit $16 \mathrm{~S}$ rDNA gene sequence. Genomic DNA for sequencing was performed using guanidinium thiocyanate method [8]. DNA sequences were determined with an automatic sequence analyzer (Abi Prismtm 3130; Applied Biosystems), using the same primers and dye terminator cycle sequencing kit (Applied Biosystems). Nearly complete $16 \mathrm{~S}$ rRNA gene sequence of approximately 1400 bases was obtained. The sequence was compared against GenBank database using BLAST, and sequences of related Nocardia type strains were retrieved from the database. Phylogenetic trees were constructed using the neighbor-joining method [9]. Topology of the trees was evaluated using bootstrap analysis of the sequence data using Mega4 software [10]. The sequencing analysis of the $16 \mathrm{~S}$ rDNA identified the organism as N. africana, based on $99 \%$ sequence similarity with the reference sequence. The obtained sequence

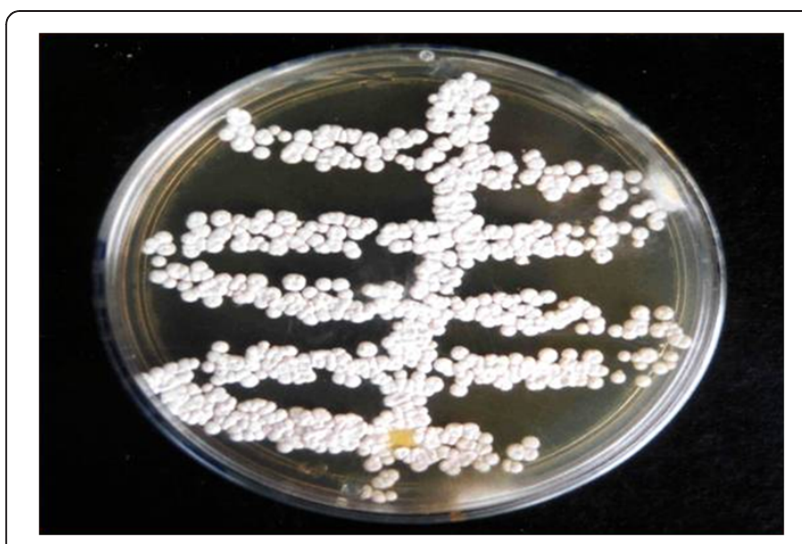

Figure 3 Whitish-orange, circular, convex, dry, with powdery surface colonies aspect of Nocardia africana in Sabouraud agar after $72 \mathrm{~h}$ of incubation, isolated from a cat with mandibular osteomyelitis. was submitted to GenBank/JJBJ/EMBL (accession No. AB636472 for the strain IFM 11168).

In vitro antimicrobial susceptibility test using the disk diffusion method [11] showed that the isolate was susceptible to trimethoprim-sulfonamide, amikacin, gentamicin, enrofloxacin, ceftiofur and amoxicillin/potassium clavulanate.

Trimethoprim-sulfonamide $(30 \mathrm{mg} / \mathrm{kg}, \mathrm{PO}, 12 \mathrm{~h}$, for 21 days), combined with fluid therapy, debridement and infusion of topical (1\%) iodine solution into the lesion were used initially in therapy. However, the clinical picture worsened, and treatment was changed to amikacin (10 mg/kg, IV, $12 \mathrm{~h}$ ) and ceftiofur (4,4 mg/kg, SC, $12 \mathrm{~h})$. After eight weeks of treatment, the animal still presented fever, weight loss, apathy, anorexia, and progressive worsening of body condition, which lead to death. Postmortem examination showed abscess formation in the submandibular lymph nodes, severe osteolysis in the left mandibular ramus, and signs of periodontitis in mandibular region of lesion. There were no lesions in other organs. Purulent secretions from the lesions were submitted for cytological, histophatological, microbiological and molecular analysis, and confirmed the diagnosis of Nocardia africana.

\section{Discussion}

Nocardiosis is an unusual clinical disease in companion animals. In cats, cutaneous-subcutaneous lesions are the main clinical sign. Usually, cutaneous-subcutaneous signs include abscesses, pustules, fistulas, cellulitis, and mycetoma, generally associated with draining of purulent secretion containing granules [12]. Our cat also presented cellulitis with draining sinuses containing granules, reinforcing the skin lesions as the most common clinical manifestation of feline nocardiosis [4].

Less frequently feline nocardiosis cause pulmonary and systemic signs [3]. Complications from cutaneoussubcutaneous nocardiosis in companion animals are represented by invasion of adjacent structures, leading to pulmonary, neurologic and/or disseminated (systemic) forms $[1,4]$. However, rarely feline nocardiosis have been associated with osteomyelitis [13]. In our cat was diagnosed uncommon mandibular osteomyelitis with intense bone proliferation and osteolysis. The transmission of microorganism in cats is associated with inhalation of soil organisms, consumption of contaminated foods, and inoculation through wounds, such as caused by territorial fights with other felids [1,14]. Despite no history of previous traumatic lesion in mandibular region or mouth in our cat, the ostemyelitis caused by Nocardia africana probably occurred secondary to periodontitis, due to ingestion of contaminated foods, or traumatic wounds in the gingiva, resembling the transmission route of nocardiosis and actinomycois in livestock [15]. 
Clinical laboratory findings in feline nocardiosis are usually nonspecific [3]. In the present report was observed leukocytosis and monocytosis, which are hematologic abnormalities observed in feline nocardiosis $[3,5]$. Severe osteolysis observed in radiographic image of mandibula in our cat was indicative of guarded prognosis, although bone infections rarely have been observed in feline nocardiosis $[3,13]$.

Traditionally, Nocardia species isolated from companion animals have been distinguished by phenotypic characteristics, based on microbiological culture, biochemical characterization, carbohydrate hydrolysis, and antimicrobial susceptibility patterns [5]. In the current report, the microorganism was isolated from samples obtained from a lesion after $72 \mathrm{~h}$ of incubation on both sheep blood agar and Sabouraud agar, reinforcing the importance of microbiological culture as a valuable method in the routine diagnosis of Nocardia.

In the present report, the isolate was initially identified as belonging to the $N$. nova complex [7]. Expanded biochemical test and antibiotic susceptibility patterns were used to differentiate members of Nocardia nova complex. Gram, Panoptico, and modified Ziehl-Neelsen stains revealed the presence of gram-positive, acid-fast, branching filamentous organisms suggestive of Nocardia sp. The histologic findings revealed a suppurative necrosis and abscesses formation usually observed in feline nocardiosis $[3,12]$. Our data highlighted the importance of combination of clinical and epidemiological data, hematologic abnormalities, radiographic images, microbiological culture, cytological and histopathological examination to improve the diagnosis of feline nocardiosis.

Sulfonamide-trimethoprim, aminoglicosides and late generation $\beta$-lactam drugs are the choices in therapy of feline nocardiosis. Successful treatment of cats with nocardiosis has been observed mainly in skin lesions $[3,12]$. In contrast, severe complications and death have been related with disseminated forms (systemic), particularly in cats co-infected with immnunosuppressive diseases $[4,16]$. In our cat, despite negative results for feline immunosuppressive diseases, therapy based on antimicrobial susceptibility testing and supportive care, the animal showed progressive worsening of body condition and died.

Recently, polymerase chain reaction, restriction fragment length polymorphisms, DNA- hybridization, and gene sequencing have enabled reclassification of some species of Nocardia. Nocardia cyriacigeorgica, Nocardia brasiliensis, Nocardia otitidiscaviarum, and Nocardia nova complex have been the most common species involved in feline nocardiosis [3]. Nocardia nova complex comprises Nocardia africana, Nocardia nova, Nocardia veterana, and Nocardia kruczakiae species based in recent molecular classification [17]. Nocardia africana infections are considered to be rare in companion animals. Nocardia africana was reported in feline mycetoma in Japan [18]. In addition, 17 cases of feline nocardiosis were described recently in Australia with involvement of Nocardia nova in cutaneous and systemic infections [4], although these cases have been diagnosed using phenotypic methods.

Currently, human nocardiosis has emerged as an increasingly common opportunistic pathogen among immunosuppressed patients, particularly those infected by AIDS, and other debilitating diseases [19]. Clinically, cutaneous-subcutaneous, pulmonary and/or neurological signs constitute the most common clinical manifestations of human nocardiosis [1]. N. asteroides, N. brasiliensis, $N$. farcinica and $N$. nova are species most frequently associated to human disease [19]. The species of Nocardia associated with human nocardiosis are similar to those most frequently found in domestic animals [5].

The main route of transmission of the genus Nocardia for people appears to be inhalation of the organism in dry warm climate regions, that facilitate the aerosolization and dispersal of pathogen, or caused by traumatic skin inoculation of organism from soil $[1,3,19]$. Microorganisms of the genus Nocardia probably are not transmitted from person-to-person form [19], and the impact of infected domestic animals in transmission of the disease to humans remains unclear [3], although cutaneoussubcutaneous infections transmitted to people by bite or scratch wounds from cats have been described elsewhere $[20,21]$. Thus, precautions should be taken by humans affected by immune dysfunction, with special attention to avoid contact with soil or organic matter contaminated by domestic animals, and management of animals suspected of nocardiosis, particularly companion animals due to close contact with humans.

\section{Conclusion}

The present report describes a rare clinical case of feline osteomyelitis caused by Nocardia africana, diagnosed based upon a combination of clinical signs, microbiological culture, cytological and histopathological findings, radiographic images, and molecular methods. The use of modern molecular techniques constitutes a valuable method for Nocardia species identification, and may contribute to identification to new species of Nocardia that are virulent in cats.

The study was approved by Ethic Commitee of College of Veterinary Medicine, Pontifícia Universidade Católica, São José dos Pinhais, State of Paraná, Brazil.

Competing interests

The authors declare that there are no conflicts of interest in this study. 


\section{Authors' contributions}

The manuscript was prepared by MGR, MRF, and LAZC, and critically discussed by other authors. Clinical examination, diagnosis and treatment of animal were performed by MRF, JW, SMR, CZC. Initial microbiological culture and characterization of the genus Nocardia was performed by KDC. Phenotypic identification and storage of isolate was carried out by LAZC and MGR. Molecular analysis of isolate was carried out by TG, TM, and KY. All authors read and approved the final manuscript.

\section{Acknowledgments}

This work was supported by Fundação de Amparo à Pesquisa do Estado de São Paulo (Fapesp), Brazil (grant number 2008/56037-1 and 2009/53494-5).

\section{Author details}

${ }^{1}$ Companion Veterinary Hospital, College of Veterinary Medicine, Pontifícia Universidade Católica, São José dos Pinhais, Parana, Brazil. ²Department of Veterinary Hygiene and Public Health, School of Veterinary Medicine and Animal Sciences, Univ. Estadual Paulista-UNESP, Botucatu, Sao Paulo, Brazil. ${ }^{3}$ Companion Animal Surgical Practice, FEPAR, Curitiba, Parana, Brazil. ${ }^{4}$ Medical Mycology Research Center, Chiba University, Chiba, Japan. ${ }^{5}$ Infectious Diseases of Domestic Animals, Department of Veterinary Hygiene and Public Health, School of Veterinary Medicine and Animal Sciences, Univ. Estadual Paulista-UNESP, Code 18618-970, POBox 560, Botucatu, Sao Paulo, Brazil.

Received: 15 July 2012 Accepted: 30 October 2012

Published: 6 December 2012

\section{References}

1. Beaman BL, Beaman L: Nocardia species: host-parasite relationships. Clin Microbiol Rev 1994, 7:213-264.

2. Quinn PJ, Carter ME, Markey B, Carter GR: Clinical Veterinary Microbiology 1994. London: Wolfe: 1994.

3. Greene CE: Infectious diseases of the dog and cat. 4th edition. St. Louis, Missouri: Elsevier; 2012

4. Malik R, Krockenberger MB, O`Brien CR, White JD, Foster D, Tisdall PLC, Gunew M, Carr PD, Bodell L, McCowan C, Howe J, Oakley C, Griffin C, Wigney DI, Martin P, Norris J, Hunt G, Mitchell DH, Gilpin C: Nocardia infections in cats: a retrospective multi-institutional study of 17 cases. Australian Veterinary J 2006, 84:235-245.

5. Ribeiro MG, Salerno T, Mattos-Guaraldi AL, Camello TCF, Langoni H, Siqueira AK, Paes AC, Fernandes MC, Lara GHB: Nocardiosis: an overview and additional report of 28 cases in cattle and dogs. Revista do Instituto de Medicina Tropical 2008, 50:177-185.

6. Kiska DL, Hicks K, Pettit DJ: Identification of medically relevant Nocardia species with an abbreviated battery of test. J Clinical Microbiology 2002, 40:1346-1351.

7. Murray PR, Baron EJ, Jorgensen JH, Pfaller MA, Yolken RH: Manual of Clinical Microbiology. Eightth edition. Washington: AMS Press; 2003.

8. Kageyama A, Torikoe N, Yazawa K, Mikami Y, Nishimura K: Nocardia asiatica sp. nov. pathogen isolated from patients with nocardiosis in Japan and clinical specimes from Thailand. Int J Syst Evol Microbiol 2004, 54:123-130

9. Saitou N, Nei M: The neighbor-joining method: a new method for reconstructing phylogenetic trees. Mol Biol Evol 1987, 4:406-425.

10. Takamura K, Dudley J, Nei M, Kumar S: MEGA4: Molecular Evolutionary Genetics Analysis (MEGA) software version 4.0. Mol Biol Evol 2007, 24:1596-1599.

11. Clinical and Laboratory Standards Institute: Performance standards for antimicrobial disk susceptibility tests. Approved standard. Eighthth edition. Wayne, USA: CLSI document M2-A9; 2006.

12. Scott W, Miller WH, Griffin CE: Small Animal Dermatology. 6th edition. Philadelphia: WB Saunders; 2001.

13. Bradney IW: Vertebral osteomyelitis due to Nocardia in a dog. Aust Vet $J$ 1985, 62:315-316.

14. Luque R, Astorga C, Tarradas B: Nocardia otitidiscaviarum infection in a cat. Vet $\operatorname{Rec} 2002,151: 488$

15. Radostits OM, Blood DC, Gay CC: Veterinary Medicine - A Textbook of the Diseases of Cattle, Sheep, Pigs, Goats and Horses. Philadelphia: Baillière Tindall; 2007.

16. Davenport DJ, Johnson GC: Cutaneous nocardiosis in a cat. J Am Vet Med Assoc 1986, 188:728-729.
17. Brown-Elliott BA, Brown JM, Conville PS, Wallace RJ: Clinical and laboratory features of the Nocardia spp. based on current molecular taxonomy. Clin Microbiol Rev 2006, 19:259-282.

18. Hattori $Y$, Kano R, Kunitani $Y$, Yanay $T$, Hasegawa A: Nocardia africana isolated from a feline mycetoma. J Clin Microbiol 2003, 41:908-910.

19. Corti ME, Villafañe-Fioti MF: Nocardiosis: a review. Int J Infect Dis 2003, 7:243-250.

20. Sachs MK: Lymphocutaneous Nocardia brasiliensis infection acquired from a cat scratch: case report and review. Clin Infect Dis 1992, 15:710-711.

21. Bottei E, Flaherty JP, Kaplan $\amalg$, Duffee-Kerr L: Lymphocutaneous Nocardia brasiliensis infection via cat scratch: a second case. Clin Infect Dis 1994, 18:649-650.

doi:10.1186/1746-6148-8-239

Cite this article as: de Farias et al:: Uncommon mandibular osteomyelitis in a cat caused by Nocardia africana. BMC Veterinary Research 2012 8:239.

\section{Submit your next manuscript to BioMed Central and take full advantage of:}

- Convenient online submission

- Thorough peer review

- No space constraints or color figure charges

- Immediate publication on acceptance

- Inclusion in PubMed, CAS, Scopus and Google Scholar

- Research which is freely available for redistribution

Submit your manuscript at www.biomedcentral.com/submit
C) Biomed Central 